\title{
The Burgeoning Role of the Gastrointestinal Microbiota
}

\author{
Martin Floch* \\ Clinical Professor of Medicine, Digestive Disease Department, USA \\ *Corresponding author: Martin Floch, Clinical Professor of Medicine, Digestive Disease Department, USA
}

\section{Short Communication}

With the release of the landmark publication containing the information that there were more microorganisms in the gastrointestinal tract than any other cells [1] the scientific community began to look at the functional role of these organisms. We edited a book on "The Microbiota in Gastrointestinal Pathophysiology; with emphasis on probiotics, prebiotics, and dysbiosis" [2] The book was published in 2018 but since that time a great deal of new information and manuscripts have appeared. Particularly in the area of probiotics and supplements containing probiotics or prebiotics so that organizations are now sponsoring national and international meetings on the subject. Until the 20th century scientific interest in the flora of the gut was largely in infectious organisms but when it was realized that the predominance of the flora in the gastrointestinal tract scientists began to look at the role of these organisms in health and disease Our book on the microbiota was divided into essentially four areas of interest. First the distribution of bacteria in the GI tract, secondly detailed microbiology on the specific bacteria used as probiotics. Thirdly on the functional role of these bacteria and fourthly on the specific literature that support these facts being related to the microbiota in these conclusions. Since publication of this text important new observations have widened the field of interest into common cancer. Colorectal cancer and colon polyps are the fourth most common cancer in the human population so that interest is intense in the western and Asian populations.

In a recent publication in Gastroenterology, the GI journal that has the highest publication impact factor, the role of the diet and bacteria have been implicated [3]. Although it has been well known as pointed out by British and African epidemiologists that a lack of dietary fiber is an important risk factor in developing colorectal cancer. Only in the past decade have bacteria of the microbiome been associated with colorectal cancer [4,3], now specifically Fusobacteria nucleatum. But much careful study and protocol has to be designed to verify the truth of this observation. The concept is so important this now is reviewed in world literature [5]. Never the less we can be certain that the role of the microbiota is pivotal in understanding immunity of the host as well as the metabolism of certain foods and now as we are finding out it is important in certain diseases, perhaps colorectal cancer, it will be exciting to health care deliverers as this information evolves. Immunity and other Disorders. The intestine is lined by intestinal epithelial cells [IEC] which act like receptors and receive impulses from bacteriologic products or any antigens in the lumen of the intestine [6] The IECs are immunological sensors to integrate appropriate bacterial, fungal, and viral signals into immunoregulatory responses. It then becomes a very complex system that involves over 13 toll like receptors [TLR] that recognize commensal or infectious responses. Antigens may present to the gut in many ways and reach the hosts including across M cells in Peyer's patches or through goblet cells. Dendritic cells [DC], recently recognized lymphocytic cells, and finally $\mathrm{T}$ cells all become involved in the immunologic response [6]. Through this very complex process the host maintains homeostatic immunologic balance or fights of infections. If It is essential for humans to maintain life in an infectious environment one has to appreciate the role of the intestine in maintaining this immunologic role.

\section{Irritable Bowel Disease [IBS]}

IBS is the most common ambulatory, gastrointestinal disorder in industrialized western and Asian societies [7] Clinicians will classify IBS into those patients with primarily diarrhea, or IBS-D, or those with constipation as IBS-C or those with both as IBSMIXED. Remembering that the definition of IBS essentially requires symptoms for at least 6 months; depending on the symptoms the treatment will vary. Patients with IBS have had success with probiotic treatment; such as Bifidobacterium infantis 35624. But the correct strain must be used. [7]. Similarly, Visbiome users 
have reported success [7]. Use in other disorders or diseases have reported successful results with use of probiotics such as ulcerative colitis, pouchitis, acute infectious illness in children and allergic disease [8] There is a growing literature on the wide use of probiotics and foods containing them.

\section{Effect on the Host Metabolism and Obesity}

Humans do not have the enzymes to metabolize short chain fatty acids [SCFA] and bile acids [BA] and numerous other enzymes to metabolize compounds essential for microbiota growth. The microbiota plays a pivotal role in basic human lipid metabolism that must be considered. This complex physiology is just being understood and is essential in human homeostasis [9]. What has become exciting has been the knowledge that obesity is related to the microbiome and can be affected by bariatric surgery [10] There is much animal study, but human studies are needed. Surely once it is fully realized that host lipid metabolism is affected by the microbiome and that this entire mechanism can be affected by bariatric surgery much human study and experimental work will evolve.

\section{References}

1. Turnbaugh PJ (2007) The human microbiome project. Nature 449: 804810.

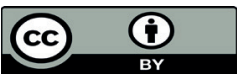

This work is licensed under Creative Commons Attribution 4.0 License

To Submit Your Article Click Here: Submit Article
2. Floch MH, Ringel Y, Walker AW (2018) The Microbiota in Gastrointestinal Pathophysiology. Elsevier ePress, pp. 442.

3. CastellarinM, WarrenRLL, FreemanJD (2012) Fusobarterium infection is prevalent in human colorectal carcinoma. Genome Res 22: 299-306.

4. LiuL Tabung FK, ZhangX (2016) Diets that promote colon inflammation associate with risk of colorectal carcinomas that contain Fusobacterium nucleatum. Clinical Gastroenterology and Hepatology 16: 1622-1631.

5. Shang FM, Liu HL (2018) Fusobacterium nucleatum and colorectal cancer: a review. World J Gastrointest Oncol 10: 71-81.

6. Madson K, Park H (2018) Immunologic response in the host in The Microbiota in Gastrointestinal Pathophysiology In: Floch, Ringel and Walker Chapter 26 Elsevier Press.

7. Hod K Ringel Y (2018) Treatment of functional gastrointestinal disorders with probiotics and prebiotics. disorders in The Mcrobiota in Gastrointestinal Pathophysiology In: Floch, Ringel, and Walker Chapter 38, Elsevier Press.

8. Floch MH, Walker WA, Sanders ME (2015) Recommendations for probiotic use : 2015 update. J Clin Gastroenterol 49(Suppl.1): S69-73.

9. SarafianMH, DingNS, HolmesE, HartA (2018) Effect on the host metabolism. In the Microbiota in Gastrointestinal Pathophysiology In: Floch MH, Ringel Y, Walker WA, chapter 28, Elsevier Press.

10. Floch N (2018) Taxanomic and metagenomic alterations of microbiota in bariatric surgery. In The microbiota in gastrointestinal pathophysiology. In: Floch, MH, Ringel Y, Walker WA Chapter 30, Elsevier Press.

$\begin{gathered}\text { Current Trends in Gastroenterology } \\ \text { and Hepatology }\end{gathered}$
Assets of Publishing with us
- Global archiving of articles
- Immediate, unrestricted online access
- Rigorous Peer Review Process
- Authors Retain Copyrights
- Unique DoI for all articles

